parallel $26^{\circ} 4$; the third and fourth comets of $188 \mathrm{I}$ and Encke's comet were also observed for position. The physical observatory at Potsdam was in full activity, and in addition to the more special subjects of observation undertaken by this important establishment, an extensive series of observations of variable stars was secured in I88I. From Stockholm Dr. Hugo Gylden notifies his determination of the parallax of the star Bradley 3077 , or No. 240 in Argelander's Catalogue of 250 stars, forming part of the seventh volume of the Bonn observations: the resulting value is $\mathrm{O}^{\prime \prime} \cdot 283 \pm \mathrm{o}^{\prime \prime} \cdot 0468$; this star has considerable proper motion. Prof. R. Wolf communicates, from Zurich, be monthly numbers of days with and without sun-spots, and the relative numbers : in the whole year's observing-days, the sun was free from spots on five days, and exhibited spots on 297.

\section{ATOMIC ATTRACTION}

THE theory of universal gravitation, as $I$ understand it, asserts

that the mutual attraction exerted by any two bodies, $\mathrm{A}$ and $B$, is dependent only on their respective masses and on the distance between them, heing entirely uninfluenced by the presence of other bodies even in the immediate neighbourhood of $\mathrm{A}$ or B. Thus at a given moment the Earth and Venus, being in certain definite positions, exert upon each other a certain force of attraction; the attraction thus taking place between the masses of the two planets would be unaltered by the removal of the Moon from the sphere of action; the gravitation of the Earth and the Moon does not therefore tie up any portion of the attractive energy of the Earth, and so diminish the force with which other bodies gravitate towards it.

A totally different assumption is usually made with regard to that form of attraction which gives rise to chemical phenomena. Here it is supposed that two or more atoms, having combined together, have thereby become incapable, at any rate in the majority of cases, of attracting others to any appreciable extent. Thus $I$ imagine that most chemists hold the view that when hydrogen and oxygen combine together to form water they thereby exhaust, or nearly exhaust, their combining power, that the power of attraction residing in the oxygen atoms is all concentrated upon the hydrogen atoms, just as we might conceive all the attractive power of the Earth concentrated on the moon, thus leaving all other bodies in its neighbourhood free from the influence of gravity. We thus invest matter with two separate forms of attraction differing entirely in their mode of action, and having indeed nothing in common. It is however possible to a certain extent to assimilate chemical attraction and gravitation, and I propose here to discuss some of the results which ensue from the elaboration of this idea. Let us suppose then that the act of chemical combination in no wise alters the power of attraction which the combining atoms exert upon surrounding bodies, and let us see what effect this bypothesis has upon the explanation of various phenomena. In order to do this we must first render as precise as possible our notions of the construction of chemical compounds.

It is now known with certainty that the atomic and molecular volumes of substances are but slightly altered by combination, that is to say, that under comparable conditions the atom of any substance generally occupies about the same space with whatever atoms, similar or dissimilar, it may be combined. This fact seems to me to point to the conclusion that the atoms which make up a molecule are as close together as their periodic motions will permit, and are not merely held in certain positions of equilibrium by various opposing forces; for if the latter supposition were true, I fail to see how it would be possible for the same atom, together with its surrounding proportion of space, to have always the same volume. The immediate proximity of the several molecules in the liquid and solid states must also be assumed, in order to account for the invariability of molecular volumes.

The innumerable facts which have been brought to light by the efforts of those who have investigated the chemistry of the carbon compounds all lead one to suppose that there is some foundation for the ideas propounded by chemists concerning the position of the atoms, and that the constitutional formula ascribed to organic substances really represent the construction of the molecule. If this be so it certainly furniches a further argument in support of the proximity of the atoms.

The assumptions contained in the preceding paragraphs are in no way opposed to the views generally held concerning molecular and atomic motion which we owe to the development of the science of heat. They merely state that there is no force of repulsion exerted between contiguous atoms, and that the vibratory or other movements are small compared with the size of the moving masses.

The object of the following remarks is to show that the hypothesis concerning chemical attraction mentioned above enables us to offer some explanation of the relative volatility of bodies. We all, I presume, look upon the maximum vapour tension of a subsiance at a given temperature as affording to a certain extent a means of estimating the attraction which its molecules exert among themselves; if there is considerable attraction there will be a low vapour tension, and with little attraction there will be a low boiling point. It f llows from this that the attraction between the molecules of hydrogen is relatively extremely small ; that in the case of oxygen and nitrogen it is also very small, though probably much larger than in the former case ; the attraction mutually exerted by molecules of chlorine will be more considerable ; while with bromine, iodine, and other liquid and solid elements it will be greater still. We must not however confound the attraction exerted between atoms of a substance with that between the molecules, for each atom attracts separately those of the contiguous molecule, so that the attraction between two molecules of bromine, for example, will be four times as great as between two atoms, and generally when the molecule of a substance contains $n$ atoms the attraction between two molecules will be approximately $n^{2}$ times that between two atoms. This is of course even approximately true only when the distance between the two molecules is great relatively to their size; when the two molecules are close together the several interatomic attractions will be exerci:ed over very different distances, and will therefore be very unequal in amount. Nevertheless, the above remark enables us to see that in some cases the apparent attraction, as estimated by the boiling-point, may be very misleading. In sulphur, for example, of which the molecule in the solid and liquid states is probably somewhat complex, we have a substance of high boiling-point, though the mutual attraction of the atoms may be comparatively small. The same is the case with carbon and many cther substances.

Applying now the above considerations to a few actual cases, w'e shall see that the relative volatility of different substances is generally satisfactorily explained. Let us designate by $(h h)$ the attraction at unit distance between two atoms of bydrogen, hy $(00)$ the attraction between two atoms of oxygen, and generally by $(r s)$ the attraction at unit distance between any two atoms, $\mathrm{R}$ and $\mathrm{S}$. Then in the case of water the molecular attraction will be represented by-

$$
4 \mathrm{~A}(h h)+{ }_{4} \mathrm{~B}(h 0)+\mathrm{C}(00)
$$

where $A, B$, and $C$ are factors dependent on the distances which separate the atoms; now we have seen that $(h h)$ and $(00)$ probably have small values, but $(h o)$ is not small, hence the attraction between molecules of water should be far greater than that between molecules of oxygen, and the boiling-point much higher, a result which is in accord with fact. The boiling-point of water would probahly be much higher than it is, were it not tha: the attractions between $\mathrm{H}$ and $\mathrm{O}$ are exerted over comparat:vely large distances, owing to the hydrogen of one molecule shieldins its companion oxygen from the approach of o! her bydrogen. Ii the similarly constituted body, $\mathrm{H}_{2} \mathrm{~S}$, the value of the molecular attraction will be-

$$
4 \mathrm{~A}(h h)+4 \mathrm{~B}(h s)+\mathrm{C}(s s)
$$

in which expression $A, B$, and $C$ may be supposed to have valnes not differing excessively from those which hold good in the case of water (the sulphuretted hydrogen being supposed liquid). The value $(s s)$ is in itself small, and since the force is exerted between two atoms which cannot approach each other very closely, $\mathrm{C}$ is also small. The affivity of hydrogen for sulphur being also feeble, the whole value of the molecular attraction is small; sulphuretted bydrogen should therefore be an extremely volatile body, which is actually the case.

With hydrochloric, hydrobıomic, and bydroidic acids we have for the molecular attraction the several values-

$$
\begin{aligned}
& \mathrm{A}(h h)+2 \mathrm{~B}(h c l)+\mathrm{C}(c l c l) \\
& \mathrm{A}^{\prime}(h h)+2 \mathrm{~B}^{\prime}(h b r)+\mathrm{C}^{\prime}(b r b r) \\
& \mathrm{A}^{\prime \prime}(h h)+2 \mathrm{~B}^{\prime \prime}(h i)+\mathrm{C}^{\prime \prime}(i i) .
\end{aligned}
$$

As the three bodies are similarly constructed we may assume that A, A', A", \&c., do not materially differ. As the third terms of these expres:ions increase the secnnd terms diminish; we should therefore expect that there might be no great difference-in the vapour-tensions of the three substances; experiment proves that 
they may be liquefied with ahout equal facility. It should be noticed that the thermal change accompanying the formation of any one of these gases, $\mathrm{HCl}$, for example, is not a true measure of the attraction between the atoms, since it also includes the heat employed in separating the atoms of the original molecules $\mathrm{H}_{2}$ and $\mathrm{Cl}_{22}$.

We may also find a confirmation of the above views in the many homologous series of organic chemistry. In the alcohols of the ethyl series, for example, the larger the molecules the greater must be the attraction between them, and consequently the higher the boiling-point; this, as is well known, is in accordance with fact. In the case of isomeric alc shols, the influence of the position of the atoms comes conspicuously to the fore. It is clear that if the atoms of carbon of two different molecules cannot approach each other so nearly in the case of one isomer as in another, the attraction between the molecules will be less, and the boiling-point consequently lower. Now in secondary and tertiary alcohols the carbon atoms are more sheltered by each other, are, as it were, more removed from the exterior of the molecule than in primary alcohols; at the same time the boiling-points are lower, which is as it should be.

If we replace two atoms of hydrogen in an alcohol by one of oxygen we increase the attraction of the molecules, since we substitute a certain number of attractions $(h o)$ and $(c o)$ for the relatively small attractions $(h h)$ and $(c h)$; the increase of boiling-point which we should expect is confirmed by experiment. Many other examples might be brought forward, were it not that their discussion would transcend the limits of this article.

Before concluding I should like to draw attention to one question which is of importance. The use of the above hypothesis renders it difficult at first sight to account for the formation of definite chemical compounds; it seems that if any number of atoms of hydrogen are equally attracted by one of chlorine, the combination of one of them with that atom would not prevent the adherence of a second and a third forming $\mathrm{H}_{2} \mathrm{Cl}, \mathrm{H}_{3} \mathrm{Cl}$, \&c. This difficulty is avoided by supposing that the chlorine atom is of such a form that only one atom of hydrogen can approach sufficiently closely to adhere permanently; such forms are difficult to imagine, though it may be remarked that an atom in the form of a ring offers in a certain sense a unique position to another which instals itself inside it. The existence of molecular compounds proves that the permanent adherence of other atoms is sometimes possible, and thus affords material support to the notion that the chemical affinity of an atom is not only exerted upon those atoms with which it is combined, but upon all others in its vicinity.

The Museum, Oxford

\section{THE GESTURE SPEECH OF MAN}

$A^{\text {NTHROPOLOGY tells the march of mankind out of }}$ savagery, in which different peoples have advanced in varying degrees, but all started in progress in civilisation from a point lower than that now occupied by the lowest of the tribes now found on earth. The marks of their rude origin, retained by all, are of the same number and kind, though differing in distinctness, showing a common origin to all intellectual and social development, notwithstanding present diversities. The most notable criterion of difference is in the copiousness and precision of oral speech, and connected with that, both as to origin and structure, is the unequal survival of gesture signs, which it is believed once universally prevailed. Where signlanguage survives it is, therefore, an instructive vestige of the prehistoric epoch, and its study may solve problems in philology and psychology. That study is best pursued by comparing the pre-eminent gesture system of the North American Indians with the more degenerate or less developed systems of other peoples.

North America showed more favourable conditions for the development of gesture signs than any other thoroughly explored part of the world. In the pré-Columbian period the population was scanty, and so subdivided dialectically that the members of but few bands could readily converse with each other. The sixty-five families of the Indian language now known to have existed within the territory of the United States differed among themselves as radically as each differed from the Hebrew, Chinese, or English. In each of these families there were sometimes as many as twenty separate languages, differing from each

${ }^{2}$ Address by Col. Garrick Mallery, U.S.A., Chairman of the Sub-Section of Anthropology at the American Association (Cincinnati). other as the Engli-h, French, German, and Persian divisions of the Aryan linguistic stock.

The conditions and circumstances attending the prevalence, and sometimes the disuse, of sign-language in North America were explained. The report of travellers, that among Indians, as well as other tribes of men, some were unable to converse in the dark, because they could not gesture, is false. It is the old story of aglossos and barbaros applied by the Greeks to all who did not speak Greek, repeated by Isaiah of the "stammering" Assyrians, and now appearing in the term slav (speaker) as contradistinguished by the Russians from the Germans, whom they stigmatise as njemez (tongueless).

The theory that sign-language was the original utterance of mankind does not depend upon such tales or prejudices. After the immeasurable period during which man has been upon the earth, it is not probable that any existing peoples can be found among whom speech has not obviated the absolute necessity for gesture in communication between themselves. The signs survive for convenience used together in oral language, and for special employment when language is unavailable.

The assertions made that the sign language of Indians originated from some one definite tribe or region supposes its comparatively recent origin, whereas the conditions favourable to its development existed very long ago and were co-extensive with the territory of North America occupied by any of the tribes. Such a solution would only be next in difficulty to the old persistent determination to decide upon the origin of the whole Indian race, in which most people of antiquity in the eastern hemisphere, including the lost tribe of Israel, the gypsies, and the Welsh, had figured conspicuously as putative parents. Numerous evidences were presented as to its antiquity and generality. But the signs are not now, and from the nature of their formation never were, identical and uniform.

An argument for the uniformity of the signs of Indians was derived from the fact that those used by any of them were generally understood by others. But signs might be understood without being identical with any before seen. There was evidence that where sign language was found among Indian tribes it had become more uniform than ever before, simply because many tribes had for some time past been forced to dwell near together at peace. The process of the formation and introduction of signs was the same among Indians as often observed among uninstructed deaf-mutes when associated together. There was a similarity of development between the sign language of mutes and Indians. The longer and closer the contact between Indians while no common tongue was adopted, the greater would be the uniformity of signs. The inference that there was but one true Indian sign language, just as there was but one true English language, was not correct, unless it could be shown that a much larger proportion of the Indians who use signs at all, than present researches show to be the case, used identically the same signs to express the same ideas, and also because the signs are not absolute and arbitrary, as are the words of English.

Are these signs conventional or instinctive? Sign language, as a product of evolution, had been developed rather than invented, and yet it seemed probable that each of the separate signs, like the several steps that lead to any true invention, had a definite origin arising out of some appropriate occasion, and th $\approx$ same sign might in this manner have had many independent origins due to identity in the circumstances, or, if lost, might have been reproduced. In regard to arbitrary or natural sounds, no signs in common use were in their origin conventional, and what appeared to be conventionality largely consisted in the form of abbreviation agreed upon. When the signs of the Indians had from ideographic become demotic, they might be called conventional, but still not arbitrary. Yet, while all Indians, as well as all gesturing men, have many signs in common, they use many others which have become conventional in the sense that their etymology and conception are not now known or regarded by those using them. The conventions by which such signs were established occurred during long periods and under many differing circumstances. Our Indians, far from being a homogeneous race and possessing uniformity in their language, religions, and customs, differ from each other more than all the several nations of Europe, and their semiotic conceptions have correspondingly differed. To insist that sign language was uniform were to assert that it is perfect. He next went on to prove the general ancient use of the system in North America. This fact might be recognised among tribes long exposed to 